УДК 639.2.081.1; 639.3; 536.4

\title{
К ТЕОРИИ ТЕРМОДИНАМИЧЕСКОГО ПОДОБИЯ УСТАНОВОК ЗАМКНУТОГО ВОДОСНАБЖЕНИЯ ДЛЯ ВЫРАЩИВАНИЯ ГИДРОБИОНТОВ
}

\author{
А. А. Недоступ, А. О. Ражев, Е. И. Хрусталев, О. М. Бедарева \\ REVISITING THE THEORY OF THERMODYNAMIC SIMILARITY OF \\ RECIRCULATING AQUACULTURE SYSTEMS FOR GROWING AQUATIC \\ ORGANISMS
}

\author{
A. A. Nedostup, A. O. Razhev, E. I. Khrustalyov, O. M. Bedareva
}

Использование установок замкнутого водоснабжения (УЗВ) требует соблюсти в них условия выращивания, приближенные к обстановке в естественном ареале обитания гидробионтов, в том числе и температурный режим. Применение для расчета поля температуры имитационного и физического моделирования позволит оптимизировать технологические процессы, увеличить продуктивность бассейнов УЗВ, повысить производительность и сократить расходы. Основной задачей термодинамического подобия установок замкнутого водоснабжения является определение силовых и объемных параметров элементов их конструкции, имеющих различные технические характеристики, что существенно усложняет теоретический анализ при проектировании УЗВ. Важная составляющая термодинамического подобия заключается в сохранении у модели, выполненной в отличных от натуры размерах, физической природы явлений оригинала. В термодинамических задачах требуется обеспечить совпадение механических и тепловых процессов. Следует привести такие зависимые от размерных признаков объекта термодинамические величины, как температура, теплота, теплоемкость, теплопроводность, температуропроводность, плотность теплового потока и другие, к их независимому безразмерному виду. При проектировании УЗВ масштабов физических характеристик гидродинамических процессов, протекающих в УЗВ при динамическом подобии, недостаточно, так как они не учитывают термодинамические процессы, протекающие в УЗВ. В статье авторами предлагаются дополнительные масштабы подобия для термодинамических характеристик, приводятся методы их вычисления и графики зависимостей от геометрического масштаба $C_{l}$. Однако необходимо понимать, что достижение полного подобия принципиально невозможно, сколь бы ни был велик перечень критериев подобия. Зная масштаб $C_{l}$, возможно определить масштабный эффект.

термодинамическое подобие, установка замкнутого водоснабжения, УЗВ

The use of recirculating aquaculture systems (RAS) requires that they observe growing conditions close to the natural habitat of hydrobionts, including the temperature regime. The use of simulation and physical modeling for the calculation of the temperature field will allow optimization of technological processes, increase the productivity of RAS basins, increase productivity and reduce costs. The main task of the 
thermodynamic similarity of recirculating aquaculture systems is to determine the power and volumetric parameters of their structural elements having different technical characteristics, which significantly complicates theoretical analysis in the design of RAS. An important component of thermodynamic similarity is preservation of the physical nature of the original phenomena in a model, executed in dimensions other than nature. In thermodynamic problems, it is required to ensure the coincidence of mechanical and thermal processes. It is necessary to characterize the interaction of the object and the medium with dimensionless quantities that would not depend on the absolute size of the object, temperature, its speed, density of the medium, viscosity of the liquid and other dimensional quantities. When designing RAS, the scale of the physical characteristics of the hydrodynamic processes occurring in the recirculating aquaculture system with dynamic similarity is not enough, as they do not take into account the thermodynamic processes occurring in the recirculating aquaculture system. In the article, the authors propose additional similarity scales for thermodynamic characteristic, give methods for their calculation and graphs of their dependences on the geometric scale $C_{l}$. It should be borne in mind that no matter how complete the list of similarity criteria is, ensuring complete similarity is fundamentally impossible, just as it is impossible to study any phenomena or processes in their entirety. Knowing the scale of $C_{l}$, it is possible to determine the scale effect.

thermodynamic similarity, recirculating aquaculture system, $R A S$

\section{ВВЕДЕНИЕ}

Ежегодно рыбоводные фермы по всему миру производят около 50 млн т рыбы. Уменьшение объемов добычи её в открытом море заставляет хозяйства переходить на более технологичные методы разведения, которые должны повысить производительность и сократить расходы [1]. Использование установок замкнутого водоснабжения требует соблюсти в них условия выращивания, приближенные к обстановке в естественном ареале обитания гидробионтов, в том числе и температурный режим. Применение для расчета поля температуры имитационного и физического моделирования позволит оптимизировать технологические процессы, увеличить продуктивность бассейнов УЗВ, повысить производительность и сократить расходы. УЗВ - это механизмы, состоящие из теплообменника, насоса, ультрафиолетовой лампы и других подсистем, которые имеют различные технические характеристики (см. рис. 1) [2]. Данный фактор существенно усложняет теоретический анализ при проектировании установок замкнутого водоснабжения, и их расчет становится невозможным. Применение УЗВ (рециркуляции воды) имеет большое значение для обеспечения продовольственной безопасности Российской Федерации. Установки замкнутого водоснабжения относятся непосредственно к рыбоводству, в том числе к техническим системам аквакультуры, поскольку хорошо приспособлены для выращивания гидробионтов. В процессе кормления в воде падает содержание кислорода, что частично компенсируется пассивным насыщением им из воздуха на поверхности воды, а частично активным процессом - оксигенацией. Выдыхаемый гидробионтами $\mathrm{CO}_{2}$, взаимодействуя с водой, уменьшает показатель $\mathrm{pH}$, увеличивая ее кислотность. Для регулирования показателя рН в УЗВ применяется аэрация, а концентрации азота в воде - частичное ее обновление посредством замкнутой системы циркуляции, которая мо- 
жет быть с биологической очисткой воды и без ее биологической очистки. Последняя менее эффективная (до 80 \%), но и менее дорогая в эксплуатации. Первая же позволяет достичь $99 \%$-ной очистки.

Эксплуатация современных установок замкнутого водоснабжения невозможна без энергозатрат. Количество потребляемой УЗВ энергии зависит как от ее производительности, так и вида выращиваемой рыбы, определяющих, в свою очередь, необходимые полезный объем и плотность посадки. В среднем суммарная энергия (тепловая и электрическая) составляет около 7 кВт·ч при плотности по-

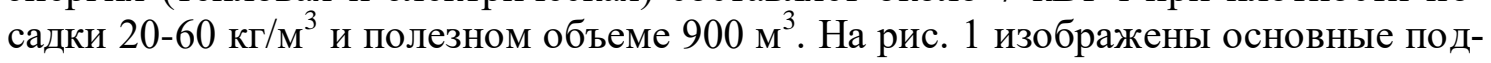
системы установки замкнутого водоснабжения для выращивания гидробионтов: бассейн; механические фильтры; оборудование или технология для денитрификации; биофильтры; насосы; обеззараживание; подогрев воды; оксигенератор.

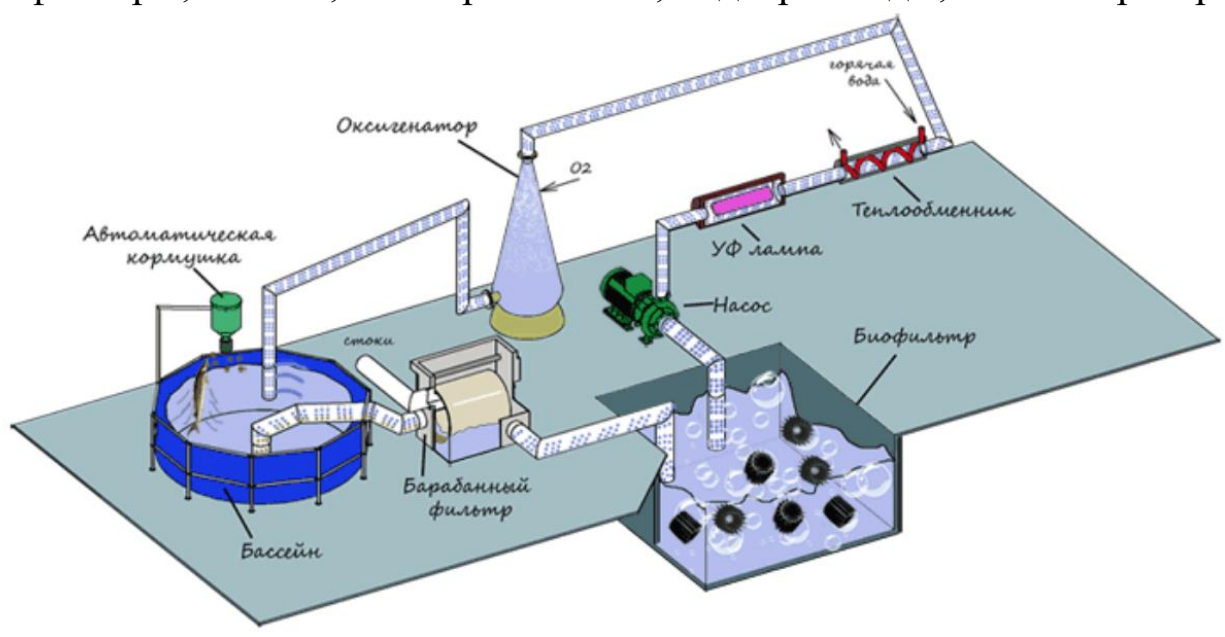

Рис. 1. Установка замкнутого водоснабжения для выращивания гидробионтов

Fig. 1. Recirculating aquaculture system for cultivation of aquatic organisms

Все указанные подсистемы крайне важны для нормальной работы УЗВ. Термодинамическое подобие как метод исследования проблем потребления воды, энергии, кислорода позволяет подобрать необходимые параметры установки замкнутого водоснабжения. Условием задачи определения термодинамического подобия применительно к модели УЗВ является сохранение физической природы явлений натурной установки замкнутого водоснабжения для основных ее подсистем, показанных на рис. 1, выполненных в отличных от натурных размерах.

\section{ПОСТАНОВКА ЗАДАЧИ}

Основной задачей термодинамического подобия установок замкнутого водоснабжения для выращивания гидробионтов является определение таких параметров УЗВ, как давление, температура и объем воды, размеры бассейнов, характеристики силовой установки [1]. Теория физического моделирования динамических процессов в данном случае имеет большое значение $[3,4]$. Необходимо, чтобы параметры модели установки замкнутого водоснабжения были геометрически подобны габаритам натурной УЗВ, применяемой в рыбоводстве. Однако модель обычно имеет отличные от натурного объекта размеры. В табл. 1 приведены основные масштабы физических характеристик гидродинамических процессов, протекающих с орудиями рыболовства при динамическом подобии. 
Таблица 1. Основные масштабы физических характеристик гидродинамических процессов, протекающих с орудиями рыболовства при динамическом подобии Table 1 . The main scale of the physical characteristics of hydrodynamic processes occurring with fishing gear with dynamic similarity

\begin{tabular}{|c|c|c|}
\hline \multicolumn{2}{|c|}{ Масштабы физических характеристик } & \multirow{2}{*}{$\begin{array}{c}\text { Преобразование } \\
\text { через масштаб } \\
C_{l}\end{array}$} \\
\hline Физические характеристики & Обозначение & \\
\hline $\begin{array}{l}\text { Геометрический параметр (длина, высо- } \\
\text { та, ширина и др.) }\end{array}$ & $C_{l}$ & $\overline{C_{l}}$ \\
\hline Геометрический параметр (площадь) & $C_{F}$ & $C_{l}^{2}$ \\
\hline Геометрический параметр (объем) & $C_{V}$ & $C_{l}^{3}$ \\
\hline Macca & $C_{m}$ & $C_{l}^{3}$ \\
\hline Время & $C_{t}$ & $C_{l}^{5 / 4}$ \\
\hline Скорость & $C_{v}$ & $C_{l}^{-1 / 4}$ \\
\hline Сила & $C_{R}$ & $C_{l}^{3 / 2}$ \\
\hline Ускорение & $C_{w}$ & $C_{l}^{-3 / 2}$ \\
\hline Объемный вес & $C_{\gamma}$ & $C_{l}^{-3 / 2}$ \\
\hline Момент & $C_{M \sigma}$ & $C_{l}^{5 / 2}$ \\
\hline Плотность & $C_{\rho}$ & 1 \\
\hline Динамическая вязкость воды & $C_{\mu}$ & $C_{l}^{3 / 4}$ \\
\hline Кинематическая вязкость воды & $C_{v}$ & $C_{l}^{3 / 4}$ \\
\hline Давление & $C_{p}$ & $C_{l}^{-1 / 2}$ \\
\hline Частота оборотов & $C_{\omega \sigma}$ & $C_{l}^{-5 / 4}$ \\
\hline Касательное напряжение & $C_{\sigma}$ & $C_{l}^{-1 / 2}$ \\
\hline Потенциальная энергия & $C_{U n}$ & $C_{l}^{5 / 2}$ \\
\hline Кинематическая энергия & $C_{T n}$ & $C_{l}^{5 / 2}$ \\
\hline Поверхностное натяжение & $C_{\sigma n}$ & $C_{l}^{1 / 2}$ \\
\hline Мощность & $C_{N}$ & $C_{l}^{5 / 4}$ \\
\hline Угол & $C_{\alpha \beta}$ & 1 \\
\hline
\end{tabular}

При проектировании установки замкнутого водоснабжения данных масштабов недостаточно, так как они не учитывают тепловые и другие процессы, протекающие в УЗВ. Возникает необходимость получить их.

\section{МЕТОДЫ}

Рассмотрим классификацию термодинамических величин [5] (см. рис. 2) и размерности основных физических характеристик термодинамики (см. табл. 2).

Найдем критерии термодинамического подобия.

Выразим масштаб количества теплоты или энтальпии, или термодинамического потенциала $L^{2} M T^{-2}$ через масштаб $C_{l}$

$$
C_{H}=C_{Q}=C_{l}^{2} C_{l}^{3} C_{l}^{-\frac{10}{4}}=C_{l}^{\frac{5}{2}} .
$$

Это справедливо, так как $C_{U n}=C_{T n}=C_{\ni}=C_{A}=C_{Q}$, где $C_{A}-$ масштаб работы; $C_{Q}$ - масштаб количества теплоты. 


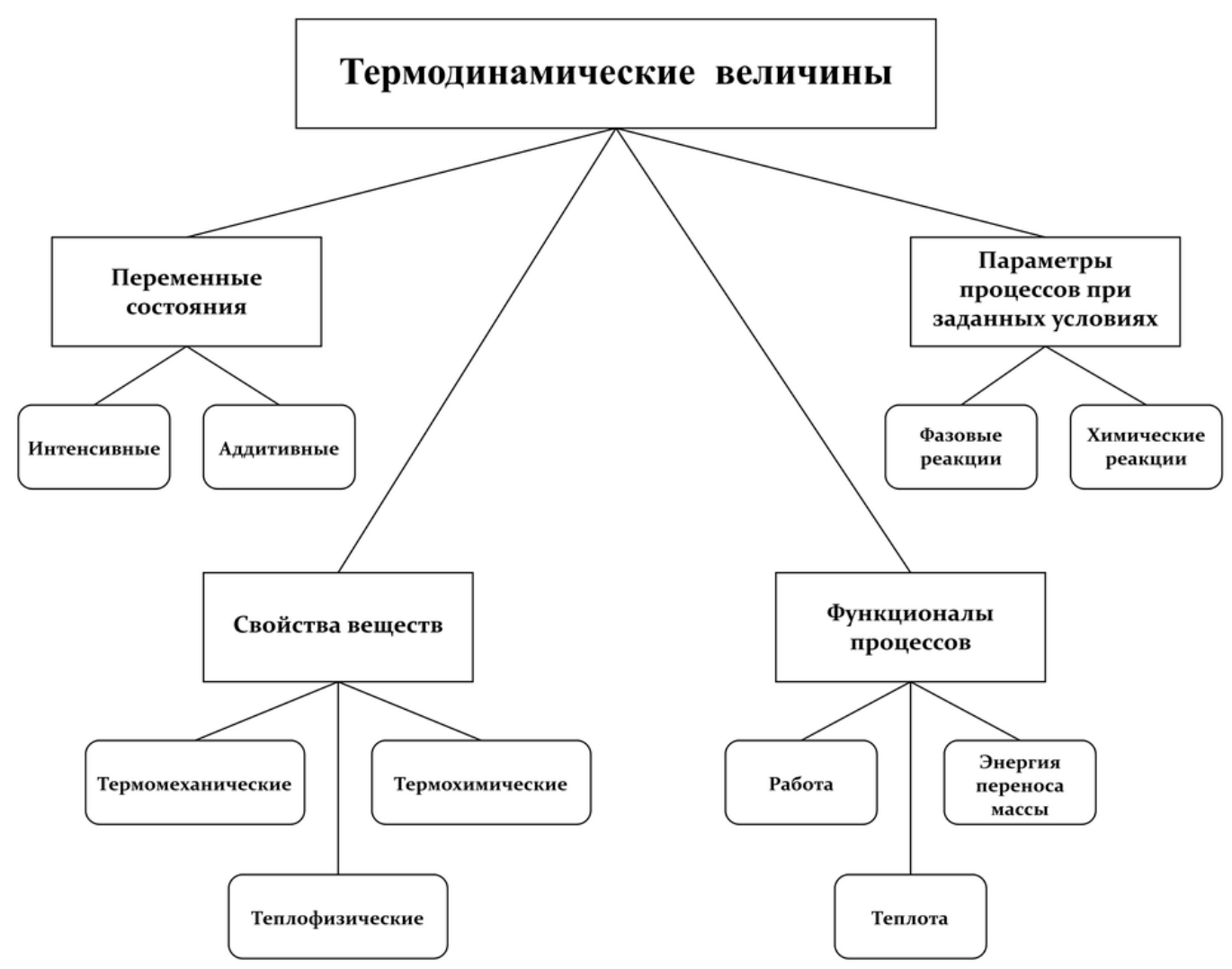

Рис. 2. Классификация термодинамических величин

Fig. 2. Classification of thermodynamic quantities

Таблица 2. Размерности основных величин термодинамики

Table 2. Dimensions of the main quantities of thermodynamics

\begin{tabular}{|l|c|}
\hline \multicolumn{1}{|c|}{ Наименование величины } & Размерность \\
\hline \multicolumn{1}{|c|}{1} & 2 \\
\hline $\begin{array}{l}\text { Количество теплоты (энтальпия), термодинамический потенциал, } \\
\text { энергия, теплота фазового превращения, теплота сгорания } \\
\text { топлива }\end{array}$ & $L^{2} M T^{2}$ \\
\hline $\begin{array}{l}\text { Удельное количество теплоты, удельный термодинамический } \\
\text { потенциал, удельная теплота химической реакции }\end{array}$ & $L^{2} T^{2}$ \\
\hline Тепловой поток & $L^{2} M T^{3}$ \\
\hline $\begin{array}{l}\text { Поверхностная плотность теплового потока, излучательная } \\
\text { способность }\end{array}$ & $M T^{3}$ \\
\hline Объемная плотность теплового потока & $L^{-1} M T^{-3}$ \\
\hline Температуропроводность & $L^{2} T^{-1}$ \\
\hline Удельный расход топлива & $L^{-2} T^{2}$ \\
\hline Температура & $K$ \\
\hline Температурный коэффициент & $K^{-1}$ \\
\hline Температурный градиент & $L^{-1} K$ \\
\hline
\end{tabular}


Окончание табл. 2

\begin{tabular}{|l|c|}
\hline \multicolumn{1}{|c|}{1} & 2 \\
\hline Теплоемкость системы, энтропия системы & $L^{2} M T^{-2} K^{-1}$ \\
\hline $\begin{array}{l}\text { Удельная теплоемкость, удельная энтропия, удельная газовая } \\
\text { постоянная }\end{array}$ & $L^{2} T^{-2} K^{-1}$ \\
\hline Объемная теплоемкость & $L^{-1} M T^{-2} K^{-1}$ \\
\hline Коэффициент лучеиспускания & $M T^{-3} K^{-4}$ \\
\hline $\begin{array}{l}\text { Коэффициент теплообмена (теплоотдачи), коэффициент теплопе- } \\
\text { редачи }\end{array}$ & $M T^{3} K^{-1}$ \\
\hline Теплопроводность & $L M T^{3} K^{-1}$ \\
\hline $\begin{array}{l}\text { Молярная внутренняя энергия, молярная энтальпия, химический } \\
\text { потенциал, химическое сродство }\end{array}$ & $L^{2} M T^{2} N^{1}$ \\
\hline Удельная молярная энтальпия & $L^{2} T^{-2} N^{-1}$ \\
\hline Молярная теплоемкость, молярная энтропия & $L^{2} M T^{-2} K^{-1} N^{-1}$ \\
\hline
\end{tabular}

Примечание. $K-$ кельвин; $N-$ количество вещества, моль.

Масштаб удельного количества теплоты

$$
C_{h}=C_{l}^{2} C_{l}^{-\frac{10}{4}}=C_{l}^{-\frac{1}{2}} .
$$

Соответственно, тепловой поток в масштабном виде

$$
C_{\Pi}=C_{l}^{2} C_{l}^{3} C_{l}^{-\frac{15}{4}}=C_{l}^{\frac{5}{4}} .
$$

Масштаб поверхностной плотности теплового потока

$$
C_{q}=C_{l}^{3} C_{l}^{-\frac{15}{4}}=C_{l}^{-\frac{3}{4}} \text {. }
$$

Выразим масштаб температуропроводности $L^{2} T^{-1}$ через масштаб $C_{l}$ :

$$
C_{\alpha}=C_{l}^{2} C_{l}^{-\frac{5}{4}}=C_{l}^{\frac{3}{4}}
$$

Масштаб объемной плотности теплового потока

$$
C_{q v}=C_{l}^{-1} C_{l}^{3} C_{l}^{-\frac{15}{4}}=C_{l}^{-\frac{7}{4}} .
$$

Удельный расход топлива в масштабном виде

$$
C_{q f u}=C_{l}^{-2} C_{l}^{\frac{10}{4}}=C_{l}^{\frac{1}{2}} \text {. }
$$

Рассмотрим формулу расчёта удельной теплоёмкости [6-8]:

$$
c=\frac{Q}{m \Delta T}
$$

где $c$ - удельная теплоёмкость; $Q$ - количество теплоты, полученное веществом при нагреве (или выделившееся при охлаждении); $m$ - масса нагреваемого (охлаждающегося) вещества; $\Delta T$ - разность конечной и начальной температур вещества.

Запишем выражение (8) в масштабном виде

$$
C_{c}=\frac{C_{Q}}{C_{m} C_{K}}=\frac{1}{C_{l}^{\frac{1}{2}} C_{K}},
$$


где $C_{c}$ - масштаб удельной теплоёмкости и энтропии системы; $C_{K}-$ масштаб разности конечной и начальной температур вещества.

Представим выражение (9) в виде

$$
C_{c} C_{K}=C_{l}^{-\frac{1}{2}} .
$$

Рассмотрим формулу расчёта полной мощности тепловых потерь [6-8]:

$$
P=-\chi \frac{S \Delta T}{l},
$$

где $P$ - полная мощность тепловых потерь; $\chi$ - коэффициент теплопроводности (удельная теплопроводность); $S$ - площадь сечения параллелепипеда; $\Delta T$ - перепад температур граней; $l$ - длина параллелепипеда, т. е. расстояние между гранями.

Запишем выражение (11) в масштабном виде

$$
C_{q v}=C_{\chi} C_{l} C_{K},
$$

где $C_{\chi}$ - масштаб коэффициентов теплопроводности (удельной теплопроводности), который равен масштабу теплопроводности.

Представим выражение (12) в виде

$$
C_{\chi} C_{K}=C_{l}^{\frac{1}{4}} .
$$

А из выражения для определения температуропроводности

$$
\alpha=\frac{\chi}{c \rho},
$$

где $\rho$ - плотность, получим масштаб температуропроводности в соответствии с выражением (9)

$$
\frac{C_{\chi} C_{K}}{C_{\rho}}=C_{l}^{\frac{1}{4}},
$$

и тогда с учетом (13) масштаб плотности $C_{\rho}=1$, что справедливо (см. табл.1).

Выражение (15) представим в виде

$$
C_{\chi} C_{K}=C_{l}^{\frac{1}{4}} \text {. }
$$

Если рассмотреть неравновесную термодинамику - раздел термодинамики, изучающий системы вне состояния термодинамического равновесия и необратимые процессы, то термодинамическая сила является разницей температур в скалярном виде $T_{1}-T_{2}=\Delta T$ (термодинамические силы порождают термодинамические потоки, как градиент температуры порождает поток теплоты). Тогда масштаб температуры представим в виде

$$
C_{K}=C_{R}=C_{l}^{\frac{3}{2}} .
$$

Масштаб коэффициента теплопроводности (удельной теплопроводности) в соответствии с (16)

$$
C_{\chi}=C_{l}^{\frac{1}{4}} C_{l}^{-\frac{3}{2}}=C_{l}^{-\frac{5}{4}} .
$$

Масштаб удельной теплоёмкости из (9)

$$
C_{c}=\frac{1}{C_{l}^{\frac{1}{2}} C_{l}^{\frac{3}{2}}}=C_{l}^{-2},
$$


Масштаб температуропроводности из (14)

$$
C_{\alpha}=\frac{C_{\chi}}{C_{c} C_{\rho}}=C_{l}^{-\frac{5}{4}} C_{l}^{2}=C_{l}^{\frac{3}{4}},
$$

так как $C_{\rho}=1$.

Масштаб температурного коэффициента (см. табл. 2)

$$
C_{\alpha \gamma}=\frac{1}{C_{K}}=C_{l}^{-\frac{3}{2}} .
$$

Масштаб температурного градиента (см. табл. 2)

$$
C_{\text {gradK }}=\frac{C_{K}}{C_{l}}=C_{l}^{\frac{3}{4}} C_{l}^{-1}=C_{l}^{-\frac{1}{4}} .
$$

На основании размерности (см. табл. 2) теплоемкости системы или энтропии системы получим их масштаб из выражения

$$
C=\frac{\delta Q}{d T}
$$

Теплоёмкость - физическая величина, определяемая как отношение количества теплоты $\delta Q$, поглощаемой/выделяемой термодинамической системой при бесконечно малом изменении её температуры $T$, к величине этого изменения $\mathrm{d} T$ :

$$
C_{C}=\frac{C_{Q}}{C_{K}}=\frac{C_{l}^{\frac{5}{2}}}{C_{l}^{\frac{3}{2}}}=C_{l} .
$$

Объемная теплоемкость

$$
c^{\prime}=\frac{C}{V} \text {. }
$$

Масштаб объемной теплоемкости (см. табл. 2)

$$
C_{c^{\prime}}=C_{l} C_{l}^{-3}=C_{l}^{-2} \text {. }
$$

Масштаб коэффициента лучеиспускания (см. табл. 2)

$$
C_{C k}=C_{l}^{3} C_{l}^{-\frac{15}{4}} C_{l}^{-\frac{12}{2}}=C_{l}^{-\frac{27}{4}} .
$$

Коэффициент теплообмена (теплоотдачи), коэффициент теплопередачи определяются по выражению [6-8]

$$
C_{\alpha}=\frac{Q}{F \Delta T},
$$

где $F$ - поверхность теплообмена, м², тогда масштаб коэффициента теплообмена (теплоотдачи) и коэффициента теплопередачи определяется как

$$
C_{C \alpha}=\frac{C_{Q}}{C_{F} C_{K}}=C_{l}^{\frac{5}{4}} C_{l}^{-2} C_{l}^{-\frac{3}{2}}=C_{l}^{-\frac{9}{4}},
$$

На основании размерностей (см. табл. 2) также можно получить масштаб коэффициента теплообмена (теплоотдачи) и коэффициента теплопередачи:

$$
C_{C \alpha}=C_{l}^{3} C_{l}^{-\frac{15}{4}} C_{l}^{-\frac{3}{2}}=C_{l}^{-\frac{9}{4}} \text {. }
$$

Теплопроводность характеризуется значением коэффициента теплопроводности (удельной теплопроводности) (18), и на основании табл. 2 


$$
C_{\chi}=C_{l} C_{l}^{3} C_{l}^{-\frac{15}{4}} C_{l}^{-\frac{3}{2}}=C_{l}^{-\frac{5}{4}}
$$

Для определения масштабов молярной внутренней энергии, молярной энтальпии, химического потенциала, химического сродства, а также масштаба дельной молярной энтальпии и масштаба молярной теплоемкости, молярной энтропии найдем соотношения масштабов из уравнения состояния идеального газа Клапейрона и уравнения Клапейрона - Менделеева.

Уравнение состояния идеального газа Клапейрона при постоянстве массы [6-8]

$$
\frac{p_{1} V_{1}}{T_{1}}=\frac{p_{2} V_{2}}{T_{2}}=\text { const },
$$

где $p_{1}, p_{2}$ - давление; $V_{1}, V_{2}$ - объем; $T_{1}, T_{2}$ - температура.

В масштабном виде уравнение (31) представим в виде

$$
\frac{C_{p} C_{V}}{C_{K}}=\frac{C_{l}^{-\frac{1}{2}} C_{l}^{3}}{C_{l}^{\frac{3}{2}}}=C_{l} .
$$

Уравнение Клапейрона - Менделеева [9]

$$
\frac{p V}{T}=\frac{m}{M} R,
$$

где $m$ - масса; $V_{1}, V_{2}$ - объем; $M$ - молярная масса; $R$ - универсальная газовая постоянная, зависящая от природы газа;

количество вещества

$$
v=\frac{m}{M} \text {. }
$$

В масштабном виде уравнение (33) представим в виде

$$
\frac{C_{p} C_{V}}{C_{K}}=\frac{C_{l}^{-\frac{1}{2}} C_{l}^{3}}{C_{l}^{\frac{3}{2}}}=\frac{C_{l}^{3}}{C_{M}} C_{k},
$$

где $C_{M}$ - масштаб молярной массы; $C_{k}$ - масштаб универсальной газовой постоянной, причем $C_{k} \neq 1$ при $C_{l} \neq 1$.

Преобразуем выражение (35) в вид

$$
C_{l}^{-2}=\frac{C_{k}}{C_{M}} .
$$

Уравнение Клапейрона - Менделеева (33) перепишем в виде

$$
p=\rho R T,
$$

тогда в масштабном виде

$$
C_{k}=C_{l}^{-2} \text {. }
$$

Из (36) и (38) видно, что масштаб молярной массы

$$
C_{M}=1 \text {. }
$$

Из (34) определим масштаб количество вещества

$$
C_{v}=C_{l}^{3} \text {. }
$$

Таким образом, справедлива формула, объединяющая основные расчёты с количеством вещества [6-9]: 


$$
n=\frac{m}{M}=\frac{N}{N_{A}}=\frac{V}{V_{m}},
$$
объем.

где $N$ - число частиц вещества; $N_{A}$ - постоянная Авогадро; $V_{m}-$ молярный

При (39) и (40) величины в (41) представим в масштабном виде

$$
\begin{aligned}
& C_{v}=C_{N}=C_{l}^{3}, \\
& C_{M}=C_{N_{A}}=C_{V_{m}}=1 .
\end{aligned}
$$

Тогда масштаб молярной внутренней энергии, молярной энтальпии, химического потенциала, химического сродства $L^{2} M T^{-2} N^{-1}$

$$
C_{U m}=C_{l}^{2} C_{l}^{3} C_{l}^{-\frac{10}{4}} C_{l}^{-3}=C_{l}^{-\frac{1}{2}},
$$

масштаб удельной молярной энтальпии

$$
C_{i}=C_{l}^{2} C_{l}^{-\frac{10}{4}} C_{l}^{-3}=C_{l}^{-4},
$$

масштаб молярной теплоемкости, молярной энтропии

$$
C_{C_{m}}=C_{l}^{2} C_{l}^{3} C_{l}^{-\frac{10}{4}} C_{l}^{-\frac{3}{2}} C_{l}^{-3}=C_{l}^{-2} .
$$

\section{РЕЗУЛЬТАТЫ И ОБСУЖДЕНИЕ}

\begin{tabular}{|c|c|c|}
\hline \multicolumn{2}{|c|}{ Масштабы физических характеристик } & \multirow{2}{*}{$\begin{array}{c}\text { Преобразование } \\
\text { через масштаб } \\
C_{l}\end{array}$} \\
\hline Физические характеристики & Обозначение & \\
\hline 1 & 2 & 3 \\
\hline Количество теплоты & $C_{Q}$ & $C_{l}^{5 / 2}$ \\
\hline Удельное количество теплоты & $C_{h}$ & $C_{l}^{-1 / 2}$ \\
\hline Тепловой поток & $C_{\Pi}$ & $C_{l}^{5 / 4}$ \\
\hline $\begin{array}{l}\text { Поверхностная плотность теплового } \\
\text { потока }\end{array}$ & $C_{q}$ & $C_{l}^{-3 / 4}$ \\
\hline Температуропроводность & $C_{\alpha}$ & $C_{l}^{3 / 4}$ \\
\hline Объемная плотность теплового потока & $C_{q v}$ & $C_{l}^{-7 / 4}$ \\
\hline Удельный расход топлива & $C_{q f u}$ & $C_{l}^{1 / 2}$ \\
\hline $\begin{array}{l}\text { Масштаб разности конечной и начальной } \\
\text { температур }\end{array}$ & $C_{K}$ & $C_{l}^{3 / 2}$ \\
\hline Удельная теплопроводность & $C_{\chi}$ & $C_{l}^{-5 / 4}$ \\
\hline Удельная теплоёмкость & $C_{c}$ & $C_{l}^{-2}$ \\
\hline Температурный коэффициент & $C_{\alpha \gamma}$ & $C_{l}^{-3 / 2}$ \\
\hline Температурный градиент & $C_{\text {gradK }}$ & $C_{l}^{-1 / 4}$ \\
\hline
\end{tabular}

С учетом масштабов термодинамики (1) - (46) отобразим основные масштабы физических характеристик термодинамического подобия в табл. 3.

Таблица 3. Основные масштабы физических характеристик термодинамического подобия

Table 3. The main scale of the physical characteristics of the thermodynamic similarity 
Окончание табл. 3

\begin{tabular}{|l|c|c|}
\hline \multicolumn{1}{|c|}{$l$} & 2 & 3 \\
\hline Теплоёмкость & $C_{C}$ & $C_{l}$ \\
\hline Объемная теплоемкость & $C_{c^{\prime}}$ & $C_{l}^{-2}$ \\
\hline Коэффициент лучеиспускания & $C_{C k}$ & $C_{l}^{-27 / 4}$ \\
\hline Коэффициент теплообмена & $C_{C \alpha}$ & $C_{l}^{-9 / 4}$ \\
\hline Универсальная газовая постоянная & $C_{k}$ & $C_{l}^{-2}$ \\
\hline Молярная масса & $C_{M}$ & 1 \\
\hline Количество вещества & $C_{\mathrm{v}}$ & $C_{l}^{3}$ \\
\hline Молярная внутренняя энергия & $C_{U m}$ & $C_{l}^{-1 / 2}$ \\
\hline Удельная молярная энтальпия & $C_{i}$ & $C_{l}^{-4}$ \\
\hline Молярная теплоемкость & $C_{C m}$ & $C_{l}^{-2}$ \\
\hline
\end{tabular}

На графиках рис. 3 и 4 покажем зависимости $C_{Q}=\mathrm{f}\left(C_{l}\right)$ и $C_{\Gamma}=\mathrm{f}\left(C_{l}\right)$.

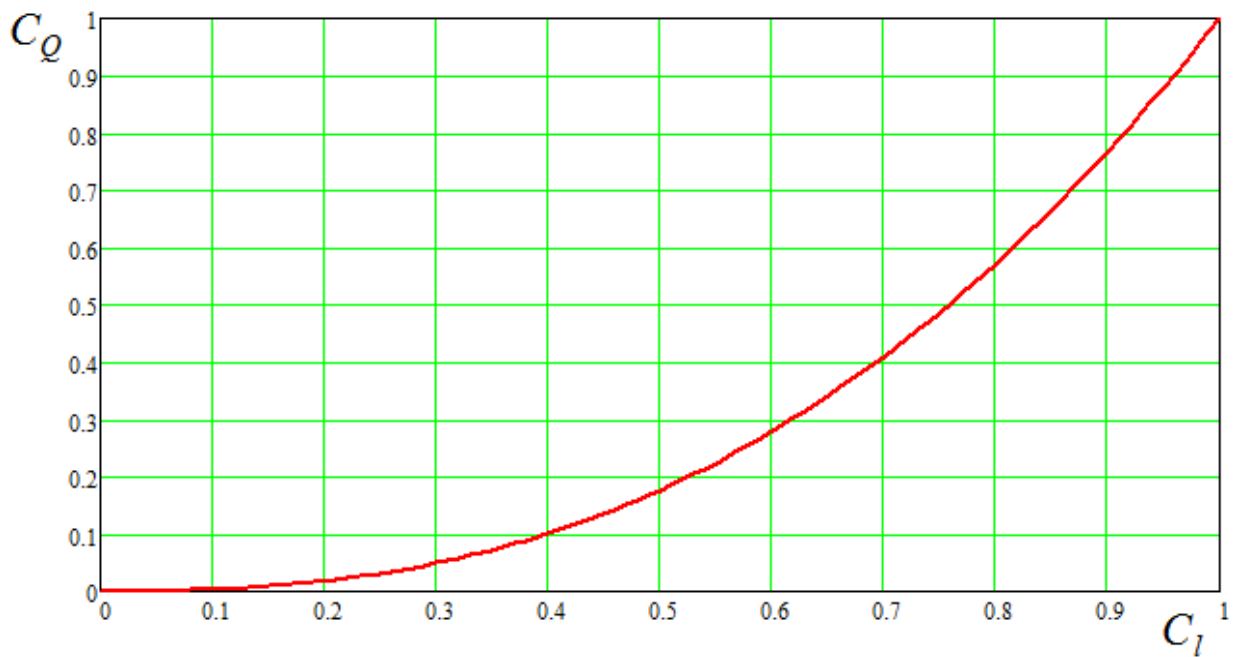

Рис. 3. График зависимости $C_{Q}=\mathrm{f}\left(C_{l}\right)$

Fig. 3. Dependence graph $C_{Q}=\mathrm{f}\left(C_{l}\right)$

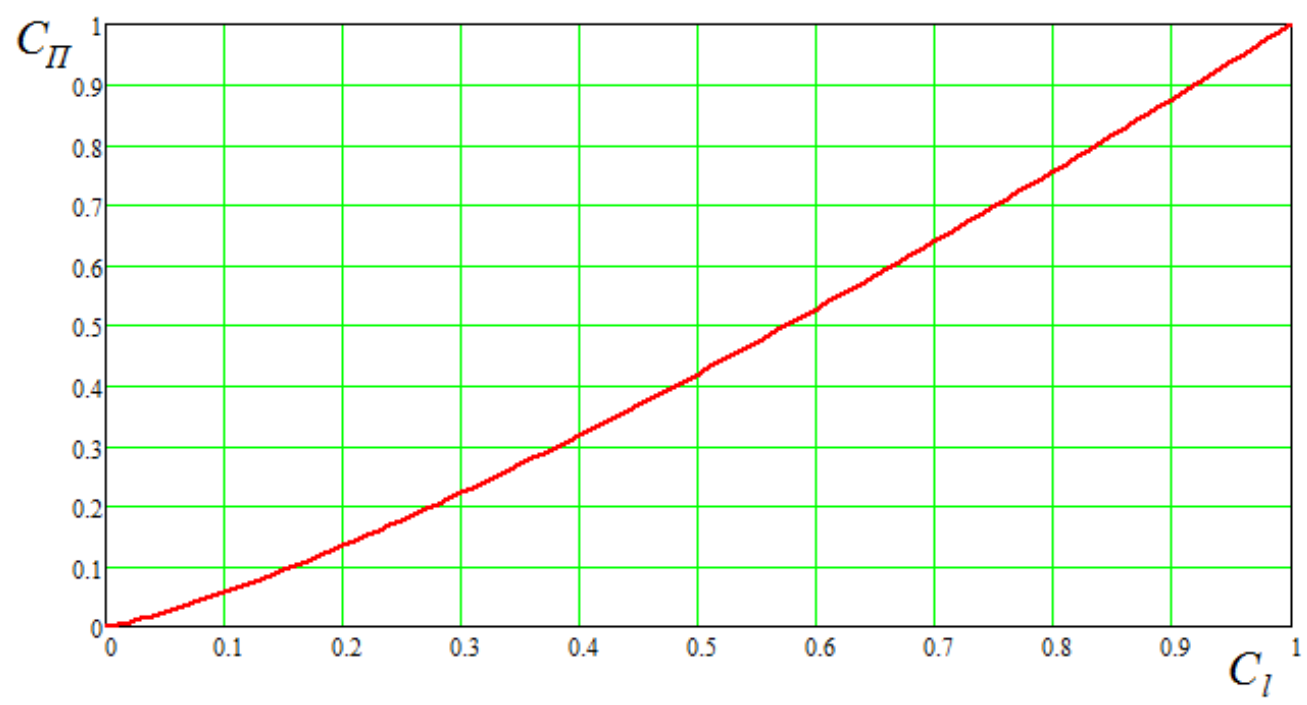

Рис. 4. График зависимости $C_{\Gamma}=\mathrm{f}\left(C_{l}\right)$

Fig. 4. Dependence graph $C_{\Pi}=\mathrm{f}\left(C_{l}\right)$ 


\section{ЗАКЛЮЧЕНИЕ}

В термодинамических задачах требуется обеспечить совпадение механических и тепловых процессов. Следует привести такие зависимые от размерных признаков объекта термодинамические величины, как температура, теплота, теплоемкость, теплопроводность, температуропроводность, плотность теплового потока и другие, к их независимому безразмерному виду. При проектировании установки замкнутого водоснабжения масштабов физических характеристик гидродинамических, грунтодинамических и трибологических процессов, протекающих с орудиями рыболовства при динамическом подобии, недостаточно, так как они не учитывают термодинамические процессы, протекающие в УЗВ. В статье авторами предлагаются дополнительные масштабы подобия для термодинамических характеристик, приводятся методы их вычисления и графики зависимостей от геометрического масштаба $C_{l}$.

\section{СПИСОК ИСПОЛЬЗОВАННЫХ ЛИТЕРАТУРНЫХ ИСТОЧНИКОВ}

1. Основы индустриальной аквакультуры: учебник / Е. И. Хрусталев, К. Б. Хайновский, О. Е. Гончаренок [и др.]. - 2-е изд., перераб. и доп. - СанктПетербург: Лань-Пресс, 2019. - 280 с.

2. Разведение рыбы в УЗВ. [Электронный ресурс]. URL: http://fish-agro.ru/fish-agro/what_about/337-razvedenie-ryby-v-uzv.html (дата обращения: 02.12.2019).

3. Недоступ, А. А. Физическое моделирование гидродинамических процессов движения орудий рыболовства / А. А. Недоступ // Вестник Томского государственного университета. Сер. Математика и механика. - Томск. - №. 3 (19). 2012. - С. 55-67.

4. Недоступ, А. А. Критерии и масштабы динамического подобия физических процессов рыболовства / А. А. Недоступ // Известия КГТУ. - № 28. - 2013. C. 227-235.

5. Термодинамика // Википедия. [Электронный ресурс]. URL:https://ru.wikipedia.org/wiki/\%D0\%A2\%D0\%B5\%D1\%80\%D0\%BC\%D0\%BE\% $\mathrm{D} 0 \% \mathrm{~B} 4 \% \mathrm{D} 0 \% \mathrm{~B} 8 \% \mathrm{D} 0 \% \mathrm{BD} \% \mathrm{D} 0 \% \mathrm{~B} 0 \% \mathrm{D} 0 \% \mathrm{BC} \% \mathrm{D} 0 \% \mathrm{~B} 8 \% \mathrm{D} 0 \% \mathrm{BA} \% \mathrm{D} 0 \% \mathrm{~B} 0 \quad$ (дата обращения: 02.12.2019).

6. Кириченко, П. А. Термодинамика, статистическая и молекулярная физика: учеб. пособие / П. А. Кириченко. - 3-е изд. - Москва: Физматкнига, 2005. - 176 с.

7. Основные принципы и понятия термодинамики. [Электронный ресурс]. URL: https://www.hvac-school.ru/upload/files/folder_48/physical-units.htm (дата обращения: 02.12.2019).

8. Базаров, И. П. Термодинамика: учебник / И. П. Базаров. - 5-е изд., стер. Санкт-Петербург: Лань, 2010. - 384 с.

9. Haynie, Donald, T. (2001). Biological Thermodynamics. Cambridge University Press. ISBN 978-0-521-79549-4. OCLC 43993556. 


\section{REFERENCES}

1. Khrustalev E. I., KHaynovskiy K. B., Goncharenok O. E., Molchanova K. A. Osnovy industrial'noy akvakul'tury: uchebnik [Fundamentals of industrial aquaculture: textbook]. SPb, Lan'-Press, 2019, vol. 2, 280 p.

2. Razvedenie ryby $v$ UZV [Fish breeding in RAS], available at: http://fish-agro.ru/fish-agro/what_about/337-razvedenie-ryby-v-uzv.html (Accessed 03 December 2019).

3. Nedostup A. A. Fizicheskoe modelirovanie gidrodinamicheskikh protsessov dvizheniya orudiy rybolovstva [Physical modeling of the hydrodynamic processes of the movement of fishing tools]. Vestnik Tomskogo gosudarstvennogo universiteta. Matematika i mekhanika. 2012, no. 3 (19), pp. 55-67.

4. Nedostup A. A. Kriterii i masshtaby dinamicheskogo podobiya fizicheskikh protsessov rybolovstva [Criteria and extent of dynamic similarity of the physical processes of fishing]. Izvestiya KGTU, 2013, no. 28, pp. 227-235.

5. Termodinamika [Thermodynamics]. Vikipediya, available at: https://ru.wikipedia.org (Accessed 03 December 2019).

6. Kirichenko P. A. Termodinamika, statisticheskaya i molekulyarnaya fizika: uchebnoe posobie [Thermodynamics, statistical and molecular physics: textbook]. Moscow, Fizmatkniga, 2005, vol. 3, 176 p.

7. Osnovnye printsipy i ponyatiya termodinamiki [Basic principles and concepts of thermodynamics], available at:

https://www.hvac-school.ru/upload/files/folder_48/physical-units.htm (Accessed 03 December 2019).

8. Bazarov I. P. Termodinamika: uchebnik [Thermodynamics: textbook]. SaintPetersburg, Lan', 2010, vol. 5, 384 p.

9. Haynie, Donald, T. (2001). Biological Thermodynamics. Cambridge University Press. ISBN 978-0-521-79549-4. OCLC 43993556.

\section{ИНФОРМАЦИЯ ОБ АВТОРАХ}

Недоступ Александр Алексеевич - Калининградский государственный технический университет; кандидат технических наук, доцент; зав. кафедрой промышленного рыболовства; E-mail: nedostup@klgtu.ru

Nedostup Alexandr Alexeevich - Kaliningrad State Technical University; $\mathrm{PhD}$ in Engineering, Associate Professor; Head of the Department of Commercial Fishery; E-mail: nedostup@klgtu.ru

Ражев Алексей Олегович - Калининградский государственный технический университет; младший научный сотрудник; E-mail: progacpp@live.ru

Razhev Alexey Olegovich - Kaliningrad State Technical University; Junior Researcher; E-mail: progacpp@live.ru 
Хрусталев Евгений Иванович - Калининградский государственный технический университет; кандидат биологических наук, доцент;

E-mail: chrustaqua@rambler.ru

Khrustalyov Evgeniy Ivanovich - Kaliningrad State Technical University; $\mathrm{PhD}$ in Biological Sciences, Associate Professor; E-mail: chrustaqua@rambler.ru

Бедарева Ольга Михайловна - Калининградский государственный технический университет; доктор биологических наук, профессор; зав. кафедрой агропочвоведения и агроэкологии; E-mail: olgabedareva@mail.ru

Bedareva Olga Mikhaylovna - Kaliningrad State Technical University; Doctor of Biological Sciences, Professor; Head of the Department of Agropedology and Agroecology; E-mail: olgabedareva@mail.ru 